\title{
Distributed Power Balancing with a Sparse Information Link
}

\author{
Wing Shing Wong and Kam Hung Lam \\ Department of Information Engineering \\ The Chinese University of Hong Kong \\ Shatin, NT, Hong Kong
}

\begin{abstract}
A new distributed power control algorithm is introduced. The algorithm is based on the assumption that some limited control data communication between the interfering transceivers is allowed. A summary of the the convergence property and simulation studies of the algorithm is presented.
\end{abstract}

\section{INTRODUCTION}

Power control is an important issue in cellular communication. Given the nature of the problem, which involves transceivers that are geographically distributed, the solution should ideally be computable by each transceiver in isolation without having to communicate with other transceivers. Unfortunately, most of the commonly known approaches to power control such as stated in [8], [4], and [2] are not completely isolated algorithms. For example, the algorithms presented in [8] and [4] allow each transceiver to compute its power level in isolation. However, the algorithms do not converge unless a common normalizing factor is chosen correctly. Hence, for these algorithms some global communication seems unavoidable in order to guarantee that the normalizing factors are chosen correctly.

In this paper, we introduce a new distributed power control algorithm. Our algorithm is based on the assumption that some limited control data communication between the interfering transceivers is al lowed. The algorithm is distributed in the sense that it is computed by each transceiver based on its local information and information sent by some of its neighbors. Of course, such information exchanges generated some overhead. However, the trade-off is that the new algorithm does not have any normalizing factor and hence, no global communication is needed. Moreover, it has a nice convergence property in the sense that the minimum of the carrier-tointerference ratios of all the interfering transceivers converge monotonically upward to a limit. Moreover, our numerical study indicates that the rate of convergence of the new algorithm is better than some of the previously proposed algorithms in the literature.
We present here only a summary of our study. Details are presented in [5]

\section{SYSTEM MODEL}

Our model is based on the model presented in [1]. There are $K$ base stations each of which has one active user. For each user there is a channel pair consisting of an uplink and a downlink channel. Propagation loss and interference effects are captured by the link gains. The notation $G_{i j}$ is used to represent the path gain received at the base station $i$ from the transmitter of the mobile unit $i$. The matrix $G=\left\{G_{i j}\right\}$ is known as the uplink gain matrix. One can define the downlink gain matrix similarly. Since the power control issue for the uplink and downlink channels are quite similar in our model, we concentrate only on uplink channel power control to simplify the discussion. Note that $G_{i i}$ represents the path gain for the intended signal, while for $i \neq j, G_{i j}$ represents the path gain for the interference signals. Thus, the $C / I$ ratio received by base station $i, \Gamma_{i}$, can be written as:

$$
\Gamma_{i}=\frac{P_{i}}{\sum_{j \neq i} Z_{i j} P_{j}}
$$

where the normalized uplink gain matrix, $Z$ is defined by $Z_{i j}=G_{i j} / G_{i i}$.

\section{CONTROL DATA FLOW STRUCTURE}

A crucial assumption in our model is that the base stations in the system are interconnected by a wired backbone network so that a small amount of control data information can be sent from one base station to another. Of course, there is a cost associated with this type of data communication and naturally one wants to minimize the cost by ensuing that this type of data communication is kept to a minimum. Hence, the algorithm we propose is designed to restrict control data traffic to network neighbors as much as possible. By network neighbors we refer to those base stations between which the control data communication cost is small.

A key concept in our distributed algorithm is the control data flow structure. This structure can be represented as a directed graph. It defines how the power 


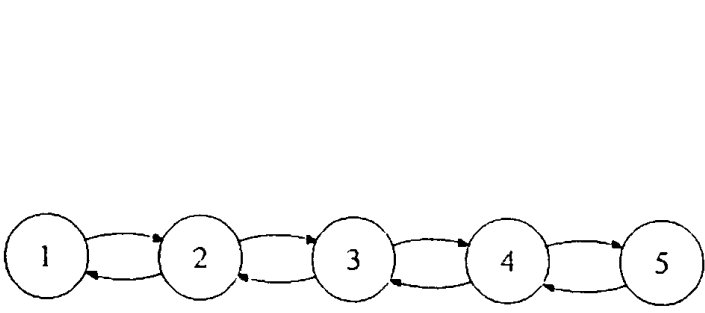

Figure 1: An example of a control data fiow structure

control data are passed among the base stations. So, if there is a directed arc from node $A$ to $B$, then control data is expected to be passed from base station $A$ to $\mathrm{B}$. The control data flow structure is dictated by the topology of the backbone network but otherwise it can be quite general except that it must satisfy the following

Reachability Condition: A control data flow structure satisfies the Reachability Condition if for any pair of nodes $(A, B)$, there is a chain of directed arcs starting from $A$ and terminating at $B$.

An example of a control data flow structure satisfying the Reachability Condition is provided in Figure 1. Since we focus on the uplink channels in this paper, the $C / I$ ratios are assumed to be measured locally at the base stations. These $C / I$ ratio measurements are averaged over a suitable time interval to smooth out fluctuations due to fast fading. At the beginning of each algorithm iteration, each base station passes the averaged $C / I$ ratio measurement to some of its neighbors according to the pre-defined control data flow structure. This information is then used in the computation of the upcoming iteration.

\section{DISTRIBUTED POWER CONTROL ALGORITHM}

The distributed power control algorithm we propose is a discrete time algorithm. At each iteration, every base station computes its own power level based on its power level in the previous iteration, its current $C / I$ ratio, and the $C / I$ ratios it receives from its neighbors. Hence, it is assumed that the computations are synchronized. The issue of asynchronous updates such as in [6] is not considered in this paper. To be more precise, the new algorithm is defined by the following set of equations:

\section{The Cooperative Algorithm}

Each mobile unit adjust its power according to the following rules:

$$
\begin{gathered}
P_{i}^{(0)}=P_{M} \\
P_{i}^{(n+1)}=\alpha_{i}^{n} P_{i}^{n} \\
\alpha_{i}^{n}=\sqrt[m]{\frac{\min \left(\Gamma_{i}^{(n)}, \max \left(\min _{j \in \mathcal{N}_{i}} \Gamma_{j}^{(n)}, \gamma_{0}\right)\right)}{\Gamma_{i}^{(n)}}}
\end{gathered}
$$

where $\mathcal{N}_{i}$ is the set of indices of base stations that send control data information to base station $i$ according to the control data flow structure, $\gamma_{0}$ is the minimum $C / I$ ratio for transmission with acceptable quality, $P_{M}$ is the maximum transmission power level of the mobile unit, and $m \geq 1$ is a parameter of the algorithm that control the rate of convergence.

The convergence properties of the Cooperative Algorithm is summarized in the following:

Theorem 1 Assume that the limiting power control vector is non-zero. Denote by $\Gamma_{i}^{*}$ the limiting $C / I$ ratio at the $i$-th base station. If $\gamma^{*} \geq \gamma_{0}$, then the Cooperative Algorithm converges to the balanced solution, that is $\Gamma_{i}^{*}=\gamma^{*}$ for all base stations. If $\gamma^{*}<\gamma_{0}$, then $\Gamma_{i}^{*} \leq \gamma_{0}$ for all base stations, with the strict inequality holding for at least one base station. Moreover, if the initial $C / I$ ratio of the $i$-th base station is larger than or equal to $\gamma_{0}$, then $\Gamma_{i}^{*}=\gamma_{0}$.

\section{NUMERICAL STUDIES}

We have carried out some simulation studies on the Cooperative Algorithm. The standard hexagonal cell layout is assumed. A fixed, homogeneous channel allocation strategy is used with a reuse factor of $N=7$. The distance between two neighboring cells, $D$, is determined by the well known formula:

$$
D=R \sqrt{3 N}
$$

where $R$ is the cell radius. $R$ is normalized to 1 , so $D=\sqrt{21}$. The base stations are assumed to be located at the center of the cells. Sixteen of the circular cells as marked in Figure 2 are assumed to use the same bandwidth and require power control to minimize the cochannel interference among them.

To simulate the users, the location of a mobile unit is generated randomly by picking a set of polar coordinate $(r, \theta)$ so that $r$ is uniformly chosen on the interval $[0,1]$ and $\theta$ is uniformly chosen from $[0,2 \pi]$. The polar coordinate is then used to define the relative position of the $i$-th mobile unit from the center of the $i$-th cell. The link gain value $G_{i j}$ is then defined by the formula:

$$
G_{i j}=\frac{A_{i j}}{d_{i j}^{4}}
$$




\begin{tabular}{|c|c|c|c|}
\hline & $\begin{array}{c}\text { within } \\
92 \%\end{array}$ & $\begin{array}{c}\text { within } \\
95 \%\end{array}$ & $\begin{array}{c}\text { within } \\
98 \%\end{array}$ \\
\hline $\begin{array}{c}\text { Coop. Alg., } \\
\text { structure } S_{1}\end{array}$ & 50.3 & 68.0 & 86.6 \\
\hline $\begin{array}{c}\text { Coop. Alg., } \\
\text { structure } S_{2}\end{array}$ & 5.3 & 7.6 & 11.7 \\
\hline Zander's Alg. & 86.6 & 154.7 & 267.7 \\
\hline
\end{tabular}

Table 1: Average number of iterations to achieve the specified target

Averaged over 1000 experiments. Mean value of balanced $C / I=17.9 \mathrm{~dB}$

where $d_{i j}$ is the distance between the $i$-th base station and the $j$-th mobile unit and $A_{i j}$ is the attenuation factor.

As we mentioned before, we only consider the effect of log-normal fading and ignore the effect of fast fading in this study. Hence, for all $i$ and $j, A_{i j}$ is log-normal distributed with

$$
\begin{gathered}
\mathrm{E}\left[10 \log _{10} A_{i j}\right]=0 d B \\
\operatorname{Var}\left[10 \log _{10} A_{i j}\right]=\sigma^{2}=(6 d B)^{2}
\end{gathered}
$$

Two types of control data flow structure are considered. Structure $S_{1}$ is represented by a directed tour in which node 1 is joined to node 2 , node 2 is joined to node 3 , and so on; (node 16 is joined to node 1.) This is one of the simplest structures possible that satisfies the Reachability Condition. In structure $S_{2}$ every cell sends its $C / I$ information to its geographical neighbors as shown in Figure 3.

A large number of simulation runs were conducted. The different rates of convergence for the different algorithms are also summarized in Table 1 which is computed based on one thousand simulation runs. In Table 2, the effects of different $m$ values are summarized. The results show that the optimal value for $m$ is 2. This confirms the theoretical result presented in the Appendix which states that for a two cell system, $m=2$ can achieve the balanced solution in one iteration.

\section{References}

[1] Aein, J. M., "Power balancing in systems employing frequency seuse," COMSAT Tech. Rev., 3(2) (1973) 277-299.

[2] Foschini, G. J., "A simple distributed autonomous power control algorithm and its convergence," to appear in IEEE Transactions on Vehicular Technology (1993).

\begin{tabular}{|c|c|}
\hline$m$ value & $\begin{array}{c}\text { Avg. \# of iterations to reach } \\
99 \% \text { of balanced value }\end{array}$ \\
\hline 1 & 76.015 \\
\hline 2 & 15.360 \\
\hline 4 & 30.045 \\
\hline 8 & 59.515 \\
\hline
\end{tabular}

Table 2: A comparison study of the effect of $m$ on the convergence rate

[3] Gantmacher, F. R., The theory of Matrices, Vol 2, Chelsea Publishing Company, New York (1959).

[4] Grandhi, S. A., R. Vijayan, D. J. Goodman, "A distributed algorithm for power control in cellular radio systems," Proc. Allerton Conference on Communicalions, Control and Computing, (1992).

[5] Lam, K. H., and W. S. Wong, "Distributed power balancing with limited control data flow," prepirnt.

[6] Mitra, D., "An asynchronous distributed algorithm for power control in cellular radio systems," Proc. Fourth WINLAB Workshop on Third Generation Wireless Information Networks (1993).

[7] Nettleton, R. W., and H. Alavi, "Power control for spread-spectrum cellular mobile radio system," Proc. IEEE Veh. Technol. Conf., (1983) 242-246.

[8] Zander, J., "Distributed cochannel interference control in cellular radio systems," IEEE Transactions on Vehicular Technology 41(3) (1992) 305311.

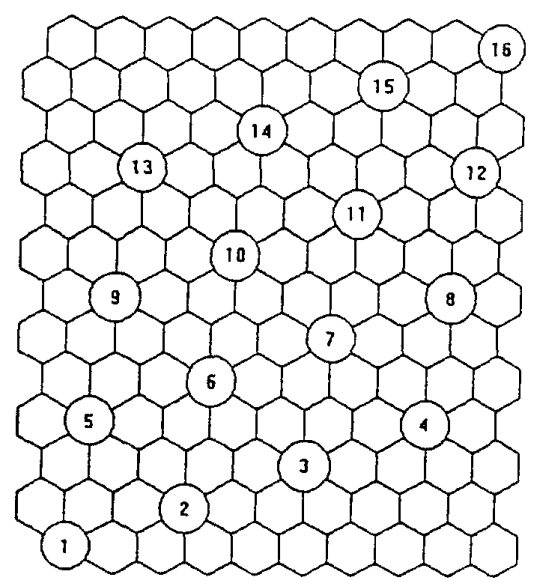

Figure 2: Layout of interfering cells in the numerical study

$$
\text { Interfering cells are assumed to be circular. }
$$



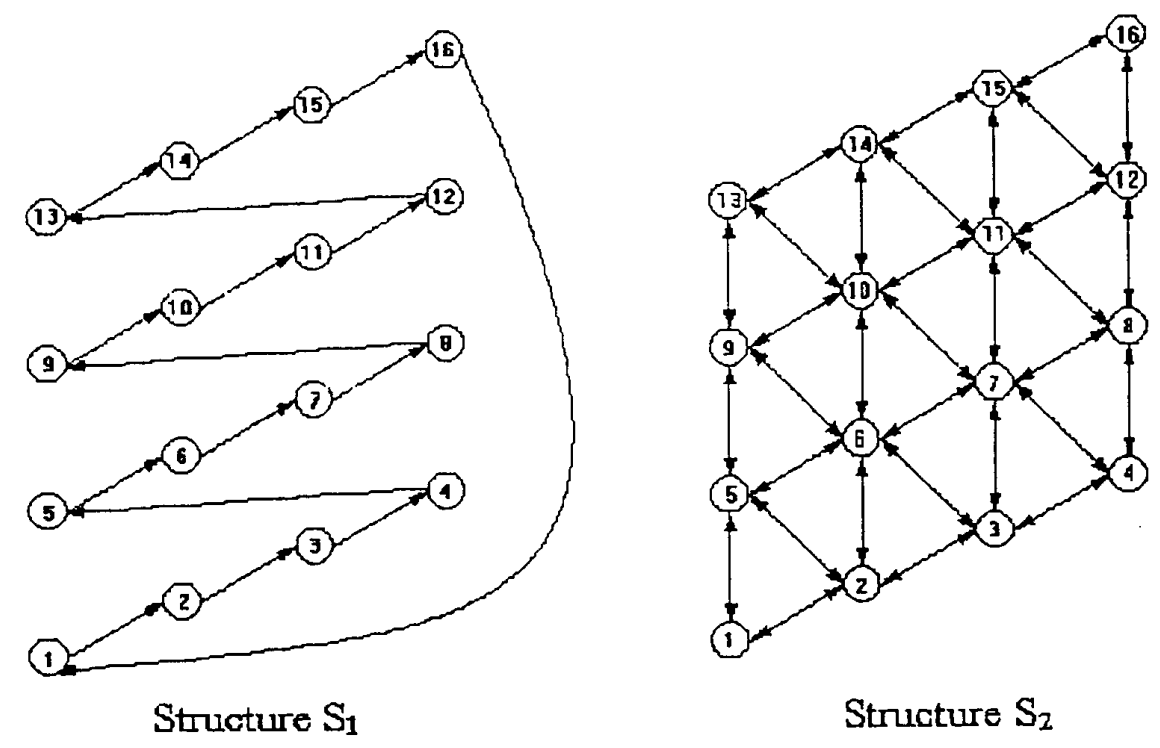

Figure 3: Data flow control structure studied in this paper 